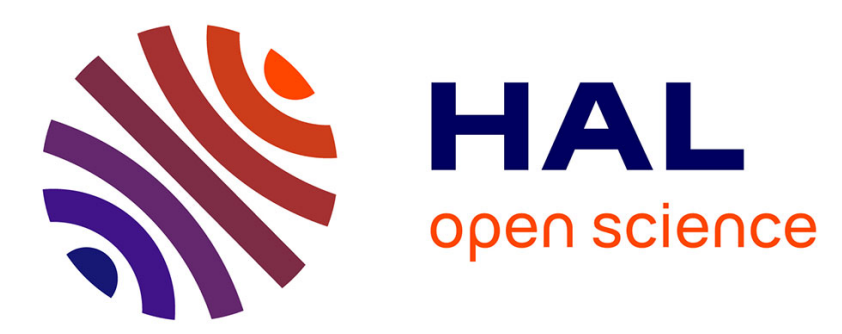

\title{
On the practical stability of hybrid control algorithm with minimum dwell-time for a DC-AC converter
}

\author{
Carolina Albea-Sanchez, Oswaldo Lopez Santos, David A. Zambrano Prada,
} Francisco Gordillo, Germain Garcia

\section{- To cite this version:}

Carolina Albea-Sanchez, Oswaldo Lopez Santos, David A. Zambrano Prada, Francisco Gordillo, Germain Garcia. On the practical stability of hybrid control algorithm with minimum dwell-time for a DC-AC converter. IEEE Transactions on Control Systems Technology, 2019, 27 (6), pp.2581-2588. 10.1109/TCST.2018.2870843. hal-01730522v2

\section{HAL Id: hal-01730522 \\ https://hal.science/hal-01730522v2}

Submitted on 17 Sep 2018

HAL is a multi-disciplinary open access archive for the deposit and dissemination of scientific research documents, whether they are published or not. The documents may come from teaching and research institutions in France or abroad, or from public or private research centers.
L'archive ouverte pluridisciplinaire HAL, est destinée au dépôt et à la diffusion de documents scientifiques de niveau recherche, publiés ou non, émanant des établissements d'enseignement et de recherche français ou étrangers, des laboratoires publics ou privés. 


\title{
On the practical stability of hybrid control algorithm with minimum dwell-time for a DC-AC converter
}

\author{
Carolina Albea Sanchez, Oswaldo Lopez Santos, David. A. Zambrano Prada, Francisco Gordillo, Germain \\ Garcia
}

\begin{abstract}
This paper presents a control law based on Hybrid Dynamical Systems (HDS) theory for a dc-ac converter. This theory is very suited for analysis of power electronic converters, since it combines continuous (voltages and currents) and discrete (on-off state of switches) signals avoiding, in this way, the use of averaged models. Here, practical stability results are induced for this tracking problem, ensuring a minimum dwell-time associated with an LQR performance level during the transient response and an admissible chattering around the operating point. The effectiveness of the resultant control law is validated by means of simulations and experiments.
\end{abstract}

\section{INTRODUCTION}

The control of power converters is characterized by the fact that the input signals are discrete, since they are the on-off state of switching devices, while the rest of signals, including the ones to be controlled, are continuous. However, most of the methods for the control of power converters use an averaged model in which the discrete signals are averaged during each sampling period and, thus, are considered continuous signals [1]-[3]. These approaches have solved many practical problems in terms of theoretical justifications, but the answers still are incomplete to some extent. Among the main limitations, we can point out the difficulty of quantifying the precision of the approximation, resulting from the averaging procedure and the fact that the properties of the control laws are only valid locally. More recently, the control community has concentrated efforts on the study of new hybrid control techniques. Indeed, Hybrid Dynamical Systems (HDS) framework is suitable for modelling and controlling this kind of systems, because it allows guaranteeing a global stability improving power converter performance, as the reduction of switching, for instance.

The HDS framework was firstly applied to electronic converters in 2003 [4] and after that more applications have appeared [5]-[7]. All these applications consider dc-dc converters, whose objective, from the control theory point of view, is to stabilize an operating point. Nevertheless, in other electronic converters is usual to work with ac and thus, the problem is more involved. When ac voltage is to be generated, as is the case of inverters, the objective is to track a sinusoidal reference. There exist some works dealing with this problem. For example, in [8], [9] authors provide a hybrid algorithm

Carolina Albea Sanchez and Germain Garcia are with LAAS-CNRS, Université de Toulouse, CNRS, Toulouse, France, e-mail: calbea,garcia@laas.fr

Oswaldo Lopez Santos and David. A. Zambrano Prad are with University of Ibagué, Colombia, e-mail: oswaldo.lopez,david.zambrano@unibague.edu.co

Francisco Gordillo is with University of Seville, Spain, e-mail: gordillo@us.es

This work has been partially funded under grants MINECO-FEDER DPI2013-41891-R and DPI2016-75294-C2-1-R. and a hybrid predictive control, respectively, for controlling a DC/AC converter with a minimum dwell-time, which is guaranteed using a space regulation. However, this dwell-time was not quantized in time for physical implementation issues. Likewise, the authors do not provide experiments to validate the controlled system.

A preliminary version of this paper appeared in [10] where a tradeoff between the switching frequency and performance was considered using a tuning parameter. In this new version, a modification of the control law is proposed in order to avoid infinitely fast switching in steady state, which is not desirable for energy efficiency and reliability considerations. Furthermore, practical stability proofs are now included. Experiments in a prototype validate the results.

The rest of the paper is organized as follows. The halfbridge inverter model is described in Sect. II while the control problem is stated in Sect. III. In Sect. IV a hybrid version of the model is proposed as well as the hybrid control law. Uniform Global Asymptotic Stability is proved. In Sect. V it is shown that one of the parameters of the control law can be used to cope with a tradeoff between dwell-time -and, thus, the switching frequency- and an LQR performance measurement. Section VI presents a modification of the control law in order to avoid infinitely fast switching at steady state. Simulations are presented in Sect. VII, and experiment results are presented in Sect. VIII. The paper closes with a section of conclusions and future work.

Notation: Through out the paper $\mathbb{R}$ denotes the set of real numbers, $\mathbb{R}^{n}$ the $\mathrm{n}$-dimensional euclidean space and $\mathbb{R}^{n \times m}$ the set of all real $n \times m$ matrices. The set of non-negative real numbers is denoted by $\mathbb{R}_{\geq 0} . M \succ 0$ (resp. $M \prec 0$ ) represents that $M$ is a symmetric positive (resp. negative) definite matrix. The Euclidan norm of vector $x \in \mathbb{R}$ is denoted by $|x| . I$ is the identity matrix of appropriate dimension. $\lambda_{m}(M), \lambda_{M}(M)$ represent the minimum and maximum eigenvalues of $M$.

\section{HALF-BRIDGE INVERTER MODEL}

Among the well-known inverter topologies used in singlephase stand-alone applications, the simpler one regarding the number of power semiconductors is the half-bridge converter. This converter feeds a resistive load $R_{0}$, from a regulated DC source $\left(2 V_{i n}\right)$ as depicted in Fig. 1. The resonant LC filter is used to increase the fundamental component amplitude, attenuating simultaneously the high frequency components. $R_{L S}$ gathers the main conduction losses.

Assumption 1. Load $R_{0}$ and voltage input $2 V_{\text {in }}$ are considered constant. We also regard $C_{1}=C_{2}$, which are large 
enough, such that the voltage ripple is negligible $\left(v_{C_{1}}=\bar{v}_{C_{1}}\right.$ and $v_{C_{2}}=\bar{v}_{C_{2}}$ ) and input voltage is symmetrically distributed among them $\left(\bar{v}_{C_{1}}=\bar{v}_{C_{2}}=V_{i n}\right)$. Therefore, the capacitor dynamics are not considered in the following. Moreover, variables $i_{L}$ and $v_{C}$ are measurable.

\section{A. System description}

The system differential equations can be written as

$$
\frac{d}{d t}\left[\begin{array}{l}
i_{L}(t) \\
v_{C}(t)
\end{array}\right]=\left[\begin{array}{cc}
-\frac{R_{L S}}{L} & -\frac{1}{L} \\
\frac{1}{C_{0}} & -\frac{1}{R_{0} C_{0}}
\end{array}\right]\left[\begin{array}{l}
i_{L}(t) \\
v_{C}(t)
\end{array}\right]+\left[\begin{array}{c}
\frac{V_{i n}}{L} \\
0
\end{array}\right] u
$$

where $i_{L}$ is the inductance current, $v_{C}$ is the capacitor voltage. Moreover, $u=U_{1}-U_{2} \in\{-1,1\}$ is the control input which represents two operating modes. The first one, $u=-1$, corresponds to $U_{1}=0\left(U_{1} \mathrm{OFF}\right)$ and $U_{2}=1\left(U_{2} \mathrm{ON}\right)$. And the second one, $u=1$, corresponds to $U_{1}=1\left(U_{1} \mathrm{ON}\right)$ and $U_{2}=0\left(U_{2} \mathrm{OFF}\right)$. Note, that due to the converter objective $v_{C}$ and $i_{L}$ are alternating voltage and current, respectively.

From a control point of view, the general control objective of this class of inverter is to stabilize system (1) in a desired oscillatory trajectory. That means, controlling the inverter such that its output voltage asymptotically tracks a sinusoidal reference.

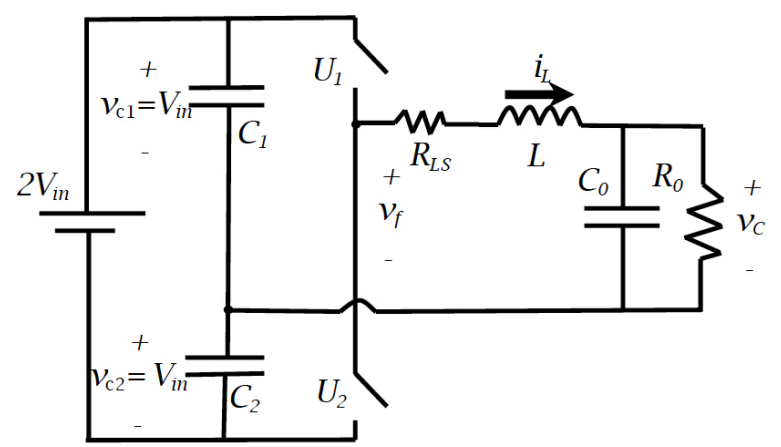

Figure 1: Half-bridge inverter.

With this goal let us consider system (1) described by the following state-space equations:

$$
\dot{x}=A x+B u
$$

where $x=\left[\begin{array}{ll}i_{L} & v_{C}\end{array}\right]^{T}$ are continuous-time variables representing the internal states, and they are assumed to be accessible, allowing to consider that $x$ is the system output. $u \in\{-1,1\}$ is the discrete-time variable corresponding to the control input. Note that $A, B$ and $C$ are matrices of appropriate dimensions.

The control objective mentioned above is to control the inverter in order to follow a desired trajectory on $v_{C}$ defined by

$$
v_{C_{d}}(t)=V_{\max } \sin (\omega t) .
$$

$V_{\text {max }}$ is the desired amplitude of the voltage through the load $R_{0}$. A simple circuit analysis shows that if in steady state $v_{C}=v_{C_{d}}$, the current $i_{L}$ in the inductance is given by

$$
i_{L_{d}}(t)=C_{0} \omega V_{\max } \cos (\omega t)+\frac{V_{\max }}{R_{0}} \sin (\omega t) .
$$

\section{PRoBlem Statement}

The objective of this work is to stabilise the half-bridge inverter in a limit cycle given by (3)-(4). To impose such a behavior, let us drive system (2) by an exogenous input $z \in \mathbb{R}^{2}$ generated by the next linear time-invariant exosystem:

$$
\dot{z}=\left[\begin{array}{cc}
0 & -\omega \\
\omega & 0
\end{array}\right] z=\Theta z \quad z(0)=\left[\begin{array}{c}
0 \\
V_{\max }
\end{array}\right] .
$$

Remark 1. From (5) it is simple to see that for all $t \in \mathbb{R}_{\geq 0}$

$$
z_{1}^{2}(t)+z_{2}^{2}(t)=V_{\max }^{2}
$$

Then, let us define the compact set

$$
\Phi=\left\{z_{1}^{2}+z_{2}^{2}=V_{\text {max }}^{2},\left(z_{1}, z_{2}\right) \in \mathbb{R}, V_{\max } \in \mathbb{R}\right\} .
$$

Note, from Remark 1, that we can reformulate our tracking problem, as a regulation problem, defining the dynamics of the overall system as

$$
\begin{aligned}
\dot{x} & =A x+B u \\
\dot{z} & =\Theta z \\
\tilde{x} & =x+D z=x-\Pi z,
\end{aligned}
$$

where $\tilde{x} \in \mathbb{R}^{2}$ is the tracking error, $D=-\Pi$ being $\Pi$ defined from (3) and (4) as:

$$
\Pi:=\left[\begin{array}{cc}
\omega C_{0} & \frac{1}{R_{0}} \\
0 & 1
\end{array}\right] .
$$

Then, from (7) and choosing

$$
\begin{aligned}
\Gamma & :=\left[\begin{array}{ll}
\Gamma_{1} & \Gamma_{2}
\end{array}\right] \\
& =\left[\frac{\omega L}{R_{0} V_{i n}}+\frac{w R_{L S} C_{0}}{V_{i n}} \quad\left(\frac{1}{L}-C_{0} \omega^{2}+\frac{R_{L S}}{L R_{0}}\right) \frac{L}{V_{i n}}\right],
\end{aligned}
$$

the tracking error dynamic is given by

$$
\begin{aligned}
\dot{\tilde{x}}=\dot{x}-\Pi \dot{z} & =A x+B u-\Pi \Theta z \\
& =A(\tilde{x}+\Pi z)+B u-\Pi \Theta z \pm B \Gamma z \\
& =A \tilde{x}+B v .
\end{aligned}
$$

being $A \Pi+B \Gamma=\Pi \Theta$ and

$$
v:=u-\Gamma z .
$$

Remark 2. Note that the algebraic equations deduced from (7) and (9)

$$
\begin{aligned}
& A \Pi+B \Gamma=\Pi \Theta . \\
& C \Pi+D=0
\end{aligned}
$$

are involved in the well-known "regulator equation", [11].

Then, let us define the compact set:

$$
\Upsilon:=\left\{v=u-\Gamma z, u \in\{-1,1\}, z \in \Phi, \Gamma \in \mathbb{R}^{1 \times 2}\right\} .
$$

Assumption 2. In (7), $v_{C_{d}}$ is supposed to be measurable as a reference in such a way that it is desired that the inverter produces a voltage with the same amplitude, frequency and phase. Then, z can be internally reconstructed, as it is shown in Fig. 2.

The aim of this work can now be stated. 


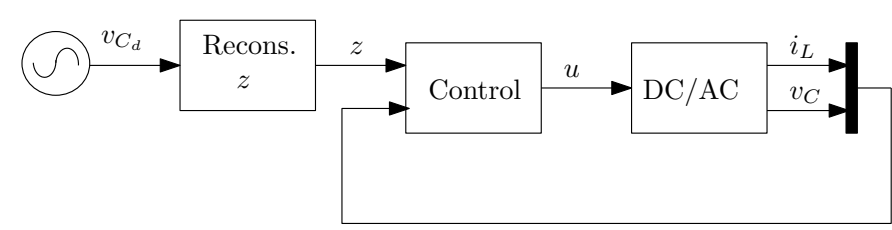

Figure 2: Block diagram of the hybrid controlled inverter.

Problem 1. Design a control law $v$ (or equivalently u) such that for any initial condition $\tilde{x}(0) \in \mathbb{R}^{2}$ :

- the trajectory of (9) is bounded,

- $\lim _{t \rightarrow \infty} \tilde{x}(t)=0$,

- a minimum dwell-time, in the transient time as well as in the steady state, is guaranteed to avoid infinite fast switching.

- it is possible to deal with the tradeoff between a minimum dwell-time and, an LQR performance level (in the transient time), as well as, with the tradeoff between a minimum dwell-time and the size of the asymptotically stable set around the compact set (6) (in the steady state).

To solve Problem 1, we use the main idea proposed in [7], formulating the problem under a hybrid dynamical framework following the theory given in [12], where continuous-time behaviour follows the evolution of the voltage and current in (1), and the discrete-time behaviour captures the switching of the control signal $u$. Likewise, we remark that the problem is an output regulation problem and the idea here is also inspired by the work in [11].

\section{HYBRID MODEL AND PROPOSED CONTROL LAW}

Let us recall that error dynamics are described by

$$
\begin{aligned}
\dot{\tilde{x}} & =A \tilde{x}+B v, \\
\dot{z} & =\Theta z,
\end{aligned}
$$

where the available input $v$ given in (10) is composed of a continuous-time signal $\Gamma z$ and a switching signal $u$, which can achieve a logical mode between 2 possible modes

$$
u \in\{-1,1\} .
$$

This paper focuses on the design of a control law for the switching signal $u$, in order to ensure suitable convergence properties of the inverter error variable $\tilde{x}$ to 0 . Following the works shown in [6], [7], [13], we represent in the following assumption a condition that characterizes the existence of a suitable switching signal inducing $\tilde{x}=0$.

Assumption 3. Given a matrix $Q \succ 0$, there exists a matrix $P \succ 0$ such that:

1) matrix $A$ verifies

$$
A^{T} P+P A+2 Q \prec 0,
$$

2) for any $z \in \Phi$, there exist $\lambda_{1, e}(t), \lambda_{-1, e}(t) \in[0,1]$ satisfying $\lambda_{1, e}+\lambda_{-1, e}=1$, such that,

$$
\lambda_{1, e}-\lambda_{-1, e}-\Gamma z=0 .
$$

Note that the solution $\tilde{x}=0$ to (13) with (10) is admissible if it is an equilibrium for the averaged system $\dot{\tilde{x}}=A \tilde{x}+$ $B\left(\lambda_{1, e}-\lambda_{-1, e}-\Gamma z\right)$. Note that the average dynamics can be perceived as the result of arbitrarily fast switching and as relaxations in the generalized sense of Krasovskii or Filippov, because (16) means that in $\tilde{x}=0$ the signal is a periodic sequence of arbitrarily small period $T$, spending a time equal to $\lambda_{1, e} T$ in mode $u=1$, and $\lambda_{-1, e} T$ in mode $u=-1$.

In order to present the proposed control law, consider the following HDS model:

$$
\mathcal{H}: \begin{cases}{\left[\begin{array}{c}
\dot{\tilde{x}} \\
\dot{z} \\
\dot{v}
\end{array}\right]} & =f(\tilde{x}, z, v), \quad(\tilde{x}, z, v) \in \mathcal{C} \\
{\left[\begin{array}{c}
\tilde{x}^{+} \\
z^{+} \\
v^{+}
\end{array}\right] \in G(\tilde{x}, z), \quad(\tilde{x}, z, v) \in \mathcal{D},} & \end{cases}
$$

where $G$ is a set-valued map representing the switching logic:

$$
\begin{aligned}
f(\tilde{x}, z, v) & :=\left[\begin{array}{c}
A \tilde{x}+B v \\
\Theta z \\
-\Gamma \Theta z
\end{array}\right] \\
G(\tilde{x}, z) & :=\left[\begin{array}{c}
\tilde{x} \\
z \\
\left(\underset{u \in\{-1,1\}}{\operatorname{argmin}} \tilde{x}^{T} P(A \tilde{x}+B(u-\Gamma z))\right)-\Gamma z
\end{array}\right]
\end{aligned}
$$

and where the flow and jump sets $\mathcal{C}$ and $\mathcal{D}$ encompass, respectively, the regions in the (extended) space $(\tilde{x}, z, v)$ where our switching strategy will continue with the current mode $v$ when the states are in set $\mathcal{C}$ or will be require to switch to a new mode when the states are in set $\mathcal{D}$. If $(\tilde{x}, z, v) \in \mathcal{D}$ then $u$ will switch according to $G$ in (18) given the value of $v=u-\Gamma z$.

Based on the parameters $P$ and $Q$ introduced in Assumption 3, we select the following flow and jump sets:

$$
\begin{aligned}
\mathcal{C} & :=\left\{(\tilde{x}, z, v): \tilde{x}^{T} P(A \tilde{x}+B v) \leq-\eta \tilde{x}^{T} Q \tilde{x}\right\} \\
\mathcal{D} & :=\left\{(\tilde{x}, z, v): \tilde{x}^{T} P(A \tilde{x}+B v) \geq-\eta \tilde{x}^{T} Q \tilde{x}\right\},
\end{aligned}
$$

where $\eta \in(0,1)$ is a useful design parameter allowing to deal with a tradeoff between an LQR performance level and switching frequency, as characterized below in Theorem 2 .

Proposition 1. The hybrid dynamical system (17)-(20) satisfies the basic hybrid conditions [12, Assumption 6.5], and then it is well-posed.

Proof. To prove the hybrid basic conditions we see that the sets $\mathcal{C}$ and $\mathcal{D}$ are closed. Moreover $f$ is a continuous function, thus it trivially satisfies outer semicontinuity and convexity properties. The map $G$ is closed, therefore it also is outer semicontiunuous [12, Lemma 5.1] and, $f$ and $G$ are locally bounded. Finally, the second conclusion of the proposition comes from [12, Theorem 6.30].

Now, we evoke Lemma in [7, Lemma 1], which is fundamental to stablish Theorem 1. 
Lemma 1. Consider that all conditions of Assumption 3 are satisfied. Then, for each $\tilde{x} \in \mathbb{R}^{2}$,

$$
\min _{u \in\{-1,1\}} \tilde{x}^{T} P(A \tilde{x}+B(u-\Gamma z)) \leq-\tilde{x}^{T} Q \tilde{x} .
$$

Proof. Define the compact set

$$
\Lambda=\left\{\lambda_{-1}, \lambda_{1} \in[0,1]: \lambda_{-1}+\lambda_{1}=1\right\} .
$$

Then, the following minimum is obtained at the extreme points:

$$
\begin{aligned}
& \min _{u \in\{-1,1\}} \tilde{x}^{T} P(A \tilde{x}+B u-B \Gamma z) \\
& =\min _{\lambda_{1}, \lambda_{-1} \in \Lambda} \tilde{x}^{T} P\left(A \tilde{x}+B \lambda_{1}-B \lambda_{-1}-B \Gamma z\right) \leq-\tilde{x}^{T} Q \tilde{x},
\end{aligned}
$$

where in the last step we used relations (15) and (16) .

The switching signals generated by our solution will depend on Lemma 1. Indeed, Assumption 3 with (19) shows that unless $\tilde{x}=\tilde{x}^{+}=0$, the solution always jumps to the interior of the flow set $\mathcal{C}$ because

$$
-\tilde{x}^{T} Q \tilde{x}<-\eta \tilde{x}^{T} Q \tilde{x},
$$

and $\eta<1$.

Following up, we will establish uniform stability and convergence properties of the hybrid system (17)-(20) to the compact attractor

$$
\mathcal{A}:=\{(\tilde{x}, z, v): \tilde{x}=0, z \in \Phi, v \in \Upsilon\},
$$

where the sets $\Phi$ and $\Upsilon$ are defined in Remark 1 and 2, respectively.

Theorem 1. Under Assumptions 2,3 the attractor (22) is uniformly globally asymptotically stable (UGAS) for hybrid system (17)-(20).

Proof. Let us take the Lyapunov function candidate

$$
V(\tilde{x})=\frac{1}{2} \tilde{x}^{T} P \tilde{x} .
$$

In the flow set, $\mathcal{C}$, using its definition in (19), we have

$$
\langle\nabla V(\tilde{x}), f(\tilde{x}, z, v)\rangle=\tilde{x}^{T} P(A \tilde{x}+B v) \leq-\eta \tilde{x}^{T} Q \tilde{x} .
$$

Across jumps we trivially get:

$$
V\left(\tilde{x}^{+}\right)-V(\tilde{x})=\frac{1}{2}\left\{\left(\tilde{x}^{+}\right)^{T} P \tilde{x}^{+}-\tilde{x}^{T} P \tilde{x}\right\}=0,
$$

because $\tilde{x}^{+}=\tilde{x}$.

Uniform global asymptotic stability is then proved applying [14, Theorem 1] to the incomplete Lyapunov function, $V$. Indeed, for a fixed $\Gamma$, which is defined in (8) and $V_{\max }, z$ and $v$ are bounded and evolve in the interior of the attractor $\mathcal{A}$, being their distances to the attractor 0 . Likewise, since the distance of $\tilde{x}$ to the attractor (22) is defined by $|\tilde{x}|=|\tilde{x}|_{\mathcal{A}}$, we have that [14, eq. (6)] holds from the structure of $V$ and from (24) and (25). [14, Theorem 1] also requires building the restricted hybrid system $\mathcal{H}_{\delta, \Delta}$ by intersecting $\mathcal{C}$ and $\mathcal{D}$ with the set

$$
S_{\delta, \Delta}=\{(\tilde{x}, z, v):|\tilde{x}| \geq \delta \quad \text { and } \quad|\tilde{x}| \leq \Delta\}
$$

and then proving (semi-global) practical persistence flow for $\mathcal{H}_{\delta, \Delta}\left(f, G, C \cap S_{\delta, \Delta}, D \cap S_{\delta, \Delta}\right)$, for each fixed values of $(\delta, \Delta)$. In particular, practical persistent flow can be guaranteed by showing that there exists $\gamma \in \mathcal{K}_{\infty}$ and $M \geq 0$, such that, all solutions to $\mathcal{H}_{\delta, \Delta}$ satisfy

$$
t \geq \gamma(j)-M, \quad \forall(t, j) \in \operatorname{dom} \xi
$$

where $\xi=(\tilde{x}, z, v)$ and

$$
\operatorname{dom} \xi=\bigcup_{j \in \operatorname{dom}_{j} \xi}\left[t_{j}, t_{j+1}\right] \times\{j\}
$$

is the hybrid time domain (see [12, Ch. 2] for details). To establish (26), notice that after each jump, from the definition of $G$ in (18) and from property (21) (in Lemma 1), we have

$$
\tilde{x}^{T} P\left(A x+B v^{+}\right) \leq-\tilde{x}^{T} Q \tilde{x}<-\eta \tilde{x}^{T} Q \tilde{x},
$$

where we used the fact that $\eta<1$ and that $(0, z, v) \notin S_{\delta, \Delta}$.

Therefore, if any solution to $\mathcal{H}_{\delta, \Delta}$ performs a jump from $S_{\delta, \Delta}$, it will remain in $S_{\delta, \Delta}$ (because $\tilde{x}$ remains unchanged) and then, from (20), it jumps to the interior of the flow set $\mathcal{C} \cap S_{\delta, \Delta}$. Moreover, from the strict inequality in (28), then all non-terminating solutions must flow for some time and since $\mathcal{C} \cap S_{\delta, \Delta}$ is bounded, there is a minimum uniform dwell-time $\rho(\delta, \Delta)$ between each pair of consecutive jumps. In particular, this dwell-time is lower-bounded by a constant, which is directly related in the state-space by an upper-bound of $V(\tilde{x})$ and $\left\langle\nabla V(\tilde{x}), f\left(\tilde{x}, z, v^{+}\right)\right\rangle$, which are $V(\tilde{x}) \leq \lambda_{m}(P) \delta^{2}$ and $\left\langle\nabla V(\tilde{x}), f\left(\tilde{x}, z, v^{+}\right)\right\rangle \leq-(1-\eta) \lambda_{m}(Q) \delta^{2}$, respectively.

This dwell-time $(\delta, \Delta)$ clearly implies [14, eq. (4)] $\gamma(j)=$ $\rho(\delta, \Delta) j$ (which is a class $\mathcal{K}_{\infty}$ function) and $M=1$. Then, all the assumptions of [14, Theorem 1] hold and UGAS of $\mathcal{A}$ is concluded.

Corollary 1. The hybrid dynamical system (17)-(20) is UGAS and is robust with respect to the presence of small noise in the state, unmodeled dynamics, and time regularization to relax the rate of switching, because the attractor (22) is compact.

Proof. From Theorem 1, we prove that the hybrid dynamic system is UGAS, and from Proposition 1 we see that it is well-posed. Moreover, as the attractor (22) is compact then it is robustly $\mathcal{K} \mathcal{L}$ asymptotically stable in a basin of attraction [12, Chapter 6].

Remark 3. Note that according to Theorem 1 system (17)(20) may exhibit a Zeno behaviour when $\tilde{x} \mapsto 0$, and consequently an infinitely fast switching may be expected, which is not acceptable in practice. This will be practically avoided later introducing an additional dwell-time logic to obtain a temporal-regularisation of the dynamics, thereby weakening asymptotic convergence into practical convergence.

Remark 4. The implementation of this control law is very simple and requires a low computational cost. The control 
law is implemented in the interior of the assigned block in Fig. 2, as follow: we initialize the constant parameters, and from the inputs, we check if the condition of the jump flow, $\mathcal{D}$ is verified. If it is the case, system jumps to the other functioning mode, because from Lemma 1 and from the fact that there are just two functioning modes, the other mode will correspond to the map of $v$ given in $G$.

\section{TRADEOFF BETWEEN DWELL-TIME AND LQR PERFORMANCE}

Once that UGAS property of the attractor is established for our solution, we focus on providing a suitable performance guarantee in order to reduce, for instance, the energy cost, current peaks and response time for the voltage or current. For this goal we apply the same paradigm shown in [7] for a hybrid context applied to switched systems.

Within the considered hybrid context, we recall that the solutions parametrized in a hybrid time domain $\operatorname{dom}_{j} \xi$ corresponds to a finite or infinite union of intervals of the form (27). Within this context, we represent a quadratic LQR performance metric focusing on flowing characteristics of the plant state, using the expression

$$
\sum_{k \in \operatorname{dom}_{j}} \int_{t_{k}}^{t_{k+1}} \tilde{x}^{T} C^{T} C \tilde{x} d \tau,
$$

where $\xi=(\tilde{x}, z, v): \operatorname{dom} \xi \rightarrow \mathbb{R}^{n} \times \mathbb{R}^{n} \times \mathbb{R}^{n}$ is a solution to hybrid system (17)-(20), for all $(t, j) \in \operatorname{dom} \xi$.

The following theorem guarantees a maximum performance cost (29) for our hybrid system.

Theorem 2. Consider hybrid system (17)-(20) satisfying Assumptions 2,3. If

$$
Q \geq I,
$$

then the following bound holds along any solution $\xi=$ $(\tilde{x}, z, v)$ of (17)-(20):

$$
J(\xi) \leq \frac{1}{2 \eta} \tilde{x}(0,0)^{T} P \tilde{x}(0,0),
$$

for all $(t, j) \in \operatorname{dom}(\xi)$.

Proof. To prove the LQR performance bound given in (31), consider any solution $\xi=(\tilde{x}, z, v)$ to $\mathcal{H}$. Then for each $(t, j) \in$ $\operatorname{dom} \xi$ and denoting $t=t_{j+1}$ to simplify notation, we have from (24)

$$
\begin{aligned}
& V(\tilde{x}(t, j))-V(\tilde{x}(0,0)) \\
& =\sum_{k=0}^{j} V\left(\tilde{x}\left(t_{k+1}, k\right)\right)-V\left(\tilde{x}\left(t_{k}, k\right)\right) \\
& =\sum_{k=0}^{j} \int_{t_{k}}^{t_{k+1}}\langle\nabla V(\tilde{x}(\tau, k)), f(\tilde{x}(\tau, k), z(\tau, k), v(\tau, k))\rangle d \tau \\
& \leq \sum_{k=0}^{j} \int_{t_{k}}^{t_{k+1}}-\eta \tilde{x}^{T}(\tau, k) Q \tilde{x}(\tau, k) d \tau \\
& \leq-\eta \sum_{k=0}^{j} \int_{t_{k}}^{t_{k+1}} \tilde{x}^{T}(\tau, k) \tilde{x}(\tau, k) d \tau
\end{aligned}
$$

where the last inequality comes from applying (30). Now, taking the limit as $t+j \rightarrow+\infty$ and using the fact that the UGAS property established in Theorem 1 implies $\lim _{t+j \rightarrow+\infty} V(\tilde{x}(t, j))=0$, we get from (32)

$$
\eta J(\xi) \leq V(\tilde{x}(0,0))=\frac{1}{2} \tilde{x}(0,0)^{T} P \tilde{x}(0,0),
$$

as to be proven.

Remark 5. Note that for a given $P$ and $Q$ that satisfy (30), the guaranteed performance level is proportional to the inverse of $\eta \in(0,1)$. That means that large values of $\eta$ are expected to drive to improved LQR performance along solutions.

On the other hand, note from the flow and jump sets in (19) and (20), that larger values of $\eta$ (close to 1) correspond to strictly larger jump sets (and smaller flow sets), which reveals that solutions with larger values of $\eta$ exhibit a larger switching frequency. In other words, through parameter $\eta$ we can deal with a tradeoff between switching frequency and an $L Q R$ performance level given in (31).

\section{A. Computation of $P$ and $Q$}

Now, we address the problem of the computation of parameters $P, Q$, following any class of optimization that reduces as much as possible the right hand side in bound (31) with any $Q \geq C^{T} C$. To this end, we select the cost function as follows [7, Section IV]:

$$
\begin{aligned}
J(\xi) & :=\min _{u} \sum_{k \in \operatorname{dom}_{j}(\xi)} \int_{t_{k}}^{t_{k+1}}\left(\frac{\rho}{R_{0}}\left(v_{C}(\tau, k)-v_{C_{d}}\right)^{2}\right. \\
& \left.+R_{L S}\left(i_{L}(\tau, k)-i_{L_{d}}\right)^{2}\right) d \tau
\end{aligned}
$$

where $\rho$ is a positive scalar. Note that the constant parameters of each term express the energy weighted sum of the error signal for each state variable. From this, we take

$$
Q=\left[\begin{array}{cc}
R_{L S} & 0 \\
0 & \frac{\rho}{R_{0}}
\end{array}\right]
$$

such that, all assumptions of Theorem 2 are satisfied.

Once $Q$ is selected, and noting that matrix $A$ is Hurwitz, the following convex optimization expressed by the linear matrix inequality always leads to a feasible solution

$$
\begin{array}{ll}
\min _{P=P^{T}>0} & \text { Trace } P, \text { subject to: } \\
& A^{T} P+P A^{T}+2 Q \prec 0,
\end{array}
$$

and this optimal solution clearly satisfies (15).

\section{PRACTICAL GLOBAL RESUlts USING TIME-REGULARIZATION}

The hybrid control law proposed above can exhibit infinitely fast switching at steady state, because given an initial condition in $\mathcal{A}$, one sees that the hybrid dynamics (17)-(20) have at least one solution that keeps jumping onto $\mathcal{A}$. Infinitely fast switching is not desirable in terms of energy efficiency and reliability, because every switch dissipates energy and reduces the switch lifespan. For this reason, we propose a few modifications of the hybrid law, aiming at reducing the 
frequency switching when $e$ is close to zero. This goal is reasonable for the proposed law, because it is possible to show that away from $\mathcal{A}$, our control law already joins a desirable property of minimum dwell time between switching, as long as Assumption 3 holds. For this, given any $v_{C_{d}}, i_{L_{d}}$ and $P, Q$ satisfying the above assumptions, consider system (17)-(20) and denote it by the shortcut notation $\mathcal{H}:=(f, G, \mathcal{C}, \mathcal{D})$ and then, for a non-negative scalar $T$ and introducing the following additional state variable $\tau$ to dynamics (17), define the following time-regularized version of $\mathcal{H}$ :

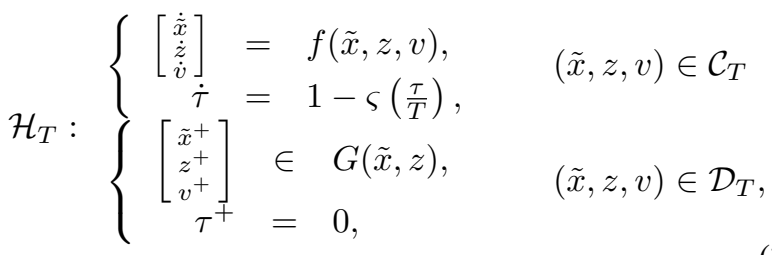

where the $\varsigma$ denotes the non-negative side of the unit deadzone function defined as $\varsigma(s):=\max \{0, s-1\}$, for all $s \geq 0$ and the jump and flow sets are the following time-regularized versions of $\mathcal{C}$ and $\mathcal{D}$ in (17)-(20):

$$
\begin{aligned}
\mathcal{C}_{T} & :=\mathcal{C} \times[0,2 T] \cup\{(\tilde{x}, z, v, \tau: \tau \in[0, T]\} \\
\mathcal{D}_{T} & :=\mathcal{D} \times[T, 2 T] .
\end{aligned}
$$

The above regularization is clearly motivated by the attempt that all solutions are forced to flow for at least $T$ ordinary time after each jump. Therefore, there are not jumps when the timer $\tau$ is too small. Note also that the deadzone given by $\varsigma$ allows a solution to flow forever while ensuring that timer $\tau$ remains in a compact set.

The introduction of the time regularization term in (34a) presents the following consequences:

- It modifies the attractor set defined in (22). Now the new attractor can be characterized by

$$
\mathcal{A}_{T}:=\{(\tilde{x}, z, v, \tau):\|\tilde{x}\| \leq \bar{X}, z \in \Phi, v \in \Upsilon, \tau \in[0,2 T]\} .
$$

- Attractor (35) is UGAS for sufficiently small values of $T>0$. Remark that attractor (35) tends to attractor (22) as $T$ tends to zero.

To show the previous statement and give an interval of values of $T$ ensuring UGAS, let us consider the following lemma [15, Theorem 3.2.2], whose proof is given for the sake of completeness.

Lemma 2. Consider Assumption 3 is satisfied, then all the eigenvalues of the matrix $P^{-1} Q$ are positive and

$$
\left\|e^{A t}\right\| \leq \frac{\lambda_{M}^{1 / 2}(P)}{\lambda_{m}^{1 / 2}(P)} e^{-\alpha t}
$$

where $\alpha=\lambda_{m}\left(P^{-1} Q\right)$.

Proof. Let us multiply (15) by $P^{-1 / 2}$ on the right and on the left side, and define $F:=P^{1 / 2} A P^{-1 / 2}$ and $\alpha:=$ $\lambda_{M}\left(P^{-1 / 2} Q P^{-1 / 2}\right)$, then we obtain:

$$
F^{T}+F \leq-2 P^{-1 / 2} Q P^{-1 / 2} \leq-2 \alpha I .
$$

Let us now define the function $\zeta(t):=\left\|e^{(F+\alpha I) t} \tilde{x}\right\|^{2}$. Then, from (36), we have

$$
\frac{d \zeta}{d t}=\tilde{x}^{T} e^{(F+\alpha I) t}\left(F^{T}+F+2 \alpha I\right) e^{(F+\alpha I) t} \tilde{x} \leq 0 .
$$

Therefore, we can remark that $\zeta$ is a non-increasing function and

$$
\left\|e^{(F+\alpha I) t} \tilde{x}\right\|^{2}=\zeta(t) \leq \zeta(0)=\|\tilde{x}\|^{2}, \quad \forall t \geq 0 .
$$

Note that this last condition can also be rewritten as

$$
\frac{\left\|e^{(F+\alpha I) t} \tilde{x}\right\|}{\|\tilde{x}\|} \leq 1
$$

which implies

$$
\left\|e^{(F+\alpha I) t}\right\| \leq 1 \Leftrightarrow\left\|e^{F t}\right\| \leq e^{-\alpha t}, \forall T \geq 0 .
$$

On the other hand, from $F$ definition and classical mathematical manipulations, we get $e^{A t}=P^{-1 / 2} e^{F t} P^{1 / 2}$. Then,

$$
\begin{aligned}
\left\|e^{A t}\right\| & =\left\|P^{-1 / 2} e^{F t} P^{1 / 2}\right\| \leq\left\|P^{-1 / 2}\right\|\left\|P^{1 / 2}\right\|\left\|e^{F t}\right\| \\
& \leq\left\|P^{-1 / 2}\right\|\left\|P^{1 / 2}\right\| e^{-\alpha t} .
\end{aligned}
$$

Note that last inequality is achieved from using (37).

Let us apply $\left\|P^{1 / 2}\right\|^{2}=\lambda_{M}(P)$ and $\left\|P^{-1 / 2}\right\|^{2}=\lambda_{m}^{-1}(P)$ which gives

$$
\left\|e^{A t}\right\| \leq \frac{\lambda_{M}^{1 / 2}(P)}{\lambda_{m}^{1 / 2}(P)} e^{-\alpha t} \quad \forall t \geq 0 .
$$

Now remark that $P^{-1 / 2} Q P^{-1 / 2}=P^{1 / 2}\left[P^{-1} Q\right] P^{-1 / 2}>$ 0 and thus, $P^{-1 / 2} Q P^{-1 / 2}$ presents the same eigenvalues as $P^{-1} Q$. Then from (36), $\alpha=\lambda_{m}\left(P^{-1 / 2} Q P^{-1 / 2}\right)=$ $\lambda_{m}\left(P^{-1} Q\right)$ and the proof is complete.

Now, we establish a useful practical minimum dwell-time property for $\mathcal{H}_{T}$.

Property 1. There exists a positive scalar $T^{*}$, such that for any chosen $T \leq T^{*}$ the solutions $(t, j) \rightarrow \xi(t, j)$ to hybrid system (34) flow for at least $T$ ordinary time after the jump before reaching set $\mathcal{D}_{T}$. Moreover, as $T$ tends to zero, the minimum dwell time tends to zero as well.

Proof. From Theorem 1, we guarantee that for all solutions far enough from $\mathcal{A}$, there exists a minimum dwell- time managed by $\eta$, as discussed in Remark 3. Consider the hybrid system (34) between two jumps in the neighbourhood of $\mathcal{A}$. Without loss of generality, consider that the first jump occurs at time $t=t_{0}$ and let us consider the variable $\tilde{t}:=t-t_{0}$. Then, in the flow set $\mathcal{C}_{T}$, the trajectories are described by:

$$
\dot{\tilde{x}}(\tilde{t})=A \tilde{x}(\tilde{t})+B u-B \Gamma z(\tilde{t}), \quad \tilde{x}(0)=\tilde{x}_{0}
$$

for a given $T>0$.

From (5), note that the trajectories of $z$ are

$$
z(\tilde{t})=\left[\begin{array}{l}
z_{1}(\tilde{t}) \\
z_{2}(\tilde{t})
\end{array}\right]=\left[\begin{array}{l}
V_{\max } \sin (\omega \tilde{t}) \\
V_{\max } \cos (\omega \tilde{t})
\end{array}\right] .
$$

Consequently, we can deduce that:

$$
\tilde{x}(\tilde{t})=e^{A \tilde{t}}\left(\tilde{x}_{0}+A^{-1} B(u-W(\tilde{t}))\right)-A^{-1} B(u-W(\tilde{t}))
$$

for $0 \leq \tilde{t} \leq T$ and with

$$
\begin{aligned}
W(\tilde{t}) & =V_{\max } \rho_{1}(\omega) \cos \left(\omega \tilde{t}+\phi_{1}(\omega)\right) \\
& +V_{\max } \rho_{2}(\omega) \sin \left(\omega \tilde{t}+\phi_{2}(\omega)\right)
\end{aligned}
$$


which is a bounded function with

$$
\begin{aligned}
\rho_{1}(\omega) & =\left|(j \omega I-A)^{-1} B \Gamma_{1}\right| \\
\rho_{2}(\omega) & =\left|(j \omega I-A)^{-1} B \Gamma_{2}\right| \\
\phi_{1}(\omega) & \left.=\arg (j \omega I-A)^{-1} B \Gamma_{1}\right) \\
\phi_{2}(\omega) & \left.=\arg (j \omega I-A)^{-1} B \Gamma_{2}\right) .
\end{aligned}
$$

Note that $\Gamma_{1}$ and $\Gamma_{2}$ come from (8).

Let rewrite (39) as

$$
\tilde{x}(\tilde{t})+A^{-1} B(u-W(\tilde{t}))=e^{A \tilde{t}}\left(\tilde{x}_{0}+A^{-1} B(u-W(\tilde{t}))\right) .
$$

From the properties of a norm, we have:

$\left\|\tilde{x}(\tilde{t})+A^{-1} B(u-W(\tilde{t}))\right\| \leq\left\|e^{A \tilde{t}}\right\|\left\|\left(\tilde{x}_{0}+A^{-1} B(u-W(\tilde{t}))\right)\right\|$

and applying Lemma 2, we get,

$$
\begin{aligned}
& \left\|\tilde{x}(\tilde{t})-A^{-1} B(u-W(\tilde{t}))\right\| \leq \\
& \frac{\lambda_{M}^{1 / 2}(P)}{\lambda_{m}^{1 / 2}(P)} e^{-\alpha \tilde{t}}\left\|\left(\tilde{x}_{0}+A^{-1} B(u-W(\tilde{t}))\right)\right\| .
\end{aligned}
$$

Now, for $0 \leq \tilde{t} \leq T$, we obtain

$$
1 \leq e^{\alpha \tilde{t}} \leq \frac{\lambda_{M}^{1 / 2}(P)}{\lambda_{m}^{1 / 2}(P)} \frac{\left\|\tilde{x}_{0}+A^{-1} B(u-W(\tilde{t}))\right\|}{\left\|\tilde{x}(\tilde{t})-A^{-1} B(u-W(\tilde{t}))\right\|}
$$

and for all $\tilde{t}>0$ and $\tilde{x}(t) \in \mathcal{C}_{T}$, we have

$$
0 \leq \tilde{t} \leq \frac{1}{\alpha} \operatorname{Ln}\left(\frac{\lambda_{M}^{1 / 2}(P)}{\lambda_{m}^{1 / 2}(P)} \frac{\left\|\tilde{x}_{0}+A^{-1} B(u-W(\tilde{t}))\right\|}{\left\|\tilde{x}(\tilde{t})-A^{-1} B(u-W(\tilde{t}))\right\|}\right) .
$$

Let us consider that the next jump occurs at time $\tilde{t}=T$, such that $(\tilde{x}(T), z(T), v(T)) \in \mathcal{D}_{T}$. Then for such $T$, we have

$$
\left\{\begin{array}{c}
\tilde{x}(\tau)^{T} P(A \tilde{x}(\tau)+B v(\tau))=-\eta \tilde{x}(\tau)^{T} Q \tilde{x}(\tau) \\
\text { and } \tau \in[T, 2 T] .
\end{array}\right.
$$

or $\backslash$ and

$$
\left\{\begin{array}{c}
\tilde{x}(\tau)^{T} P(A \tilde{x}(\tau)+B v(\tau)) \leq-\eta \tilde{x}(\tau)^{T} Q \tilde{x}(\tau) \\
\text { and } \tau=T .
\end{array}\right.
$$

Consequently, there exists a maximum dwell time bound defined by $T^{*}$ which satisfies:

$$
T^{*}=\frac{1}{\alpha} \operatorname{Ln}\left(\frac{\lambda_{M}^{1 / 2}(P)}{\lambda_{m}^{1 / 2}(P)} \frac{\left\|\tilde{x}_{0}+A^{-1} B\left(u-W\left(T^{*}\right)\right)\right\|}{\left\|\tilde{x}\left(T^{*}\right)-A^{-1} B\left(u-W\left(T^{*}\right)\right)\right\|}\right),
$$

such that,

$$
0 \leq T \leq T^{*}
$$

Note that $\tilde{x}_{0}$ and $\tilde{x}(T)$ define the maximum possible chattering in the system induced by the dwell time bound, $T^{*}$.

Property 1 ensures that some minimum dwell-time is guaranteed if solutions remain sufficiently far from $\mathcal{A}$. Moreover, using this property, the following desirable results are enjoyed by hybrid system $\mathcal{H}_{T}$.

Theorem 3. Consider $a \lambda_{1}, \lambda_{-1}$ and matrices $P \succ 0$ and $Q \succ 0$ satisfying Assumption 3. The following holds:

1) all solutions to $\mathcal{H}_{T}$ enjoy a minimum dwell-time property with a minimum $T$;
2) set $\mathcal{A}_{T}$ in (35) is compact for any positive scalar $T \leq$ $T^{*}$.

3) for any positive scalar $T \leq T^{*}$, set $\mathcal{A}_{T}$ in (35) is UGAS for dynamics $\mathcal{H}_{T}$ in (34);

4) set $\mathcal{A} \times\{0\}$ is globally practically asymptotically stable for (34), with respect to parameter $T$ (namely as long as $T$ is sufficiently small, the UGAS set $\mathcal{A}_{T}$ in (35) can be made arbitrarily close to $\mathcal{A} \times\{0\}$.

Proof. Note that hybrid system (34) enjoys the hybrid basic conditions of [12, As. 6.5], because sets $\mathcal{C}_{T}$ and $\mathcal{D}_{T}$ are both closed and $f$ and $G$ enjoy desirable properties. Then we may apply several useful results pertaining well-posed hybrid systems (specifically, in the proof of item 2 below).

Proof of item 1 . Note that the solutions present a minimum dwell-time property because the jumps are forbidden, at least, until the timer variable $\tau$ has reached the value $T$. Since $\dot{\tau}=1$ for all $\tau \leq T$, then all solutions flow for at least $T$ ordinary time after each jump (because $\tau^{+}=0$ across jumps).

Proof of item 2. Note that the hybrid system (34) satisfies Property 1 . From this property is easy to see that $\tilde{x}$ is bounded in the flow set $\mathcal{C}_{T}$. Indeed, from (39) and the properties of a norm, we have

$\|\tilde{x}(\tilde{t})\| \leq\left\|e^{A \tilde{t}}\right\|\left\|\left(\tilde{x}_{0}+A^{-1} B(u-W(\tilde{t}))\right)\right\|+\left\|A^{-1} B(u-W(\tilde{t}))\right\|$

and applying Lemma 2 and the variable change $\tilde{t}=t-t_{0}$, we obtain

$$
\begin{aligned}
\|\tilde{x}(t)\| & \leq \frac{\lambda_{M}^{1 / 2}(P)}{\lambda_{m}^{1 / 2}(P)} e^{-\alpha t}\left\|\left(\tilde{x}_{0}+A^{-1} B(u-W(t))\right)\right\| \\
& +\left\|A^{-1} B(u-W(t))\right\|:=\bar{X}, \quad \forall t \geq t_{0},
\end{aligned}
$$

proving attractor $\mathcal{A}_{T}$ is compact.

Proof of item 3. From $V(\tilde{x})$ given in (23), and the bound given before $\|\tilde{x}(t)\| \leq \bar{X}$, consider the following Lyapunov function candidate:

$$
V_{T}(\tilde{x})=\max \left\{V(\tilde{x})-\lambda_{m}(P) \bar{X}^{2}, 0\right\}
$$

which is clearly positive definite with respect to $\mathcal{A}_{T}$ and radially unbounded. Since outside set $\mathcal{A}_{T}$ the hybrid dynamics $\mathcal{H}_{T}$ coincides with the one of $\mathcal{H}$, then equations (24) and (25) hold for any $(\tilde{x}, z, v)$ not in $\mathcal{A}_{T}$, which implies that

$$
\begin{array}{cr}
\left\langle\nabla V_{T}(\tilde{x}), f(\tilde{x}, z, v)\right\rangle<0 & \forall \tilde{x} \in \mathcal{C}_{T} \backslash \mathcal{A}_{T} \\
V_{T}\left(\tilde{x}^{+}\right)-V_{T}(\tilde{x})=0, & \forall \tilde{x} \in \mathcal{D}_{T} \backslash \mathcal{A}_{T} .
\end{array}
$$

Moreover, from the property established in item 1, all complete solutions to $\mathcal{H}_{T}$ must flow for some time, and therefore from (41), we have that no solution can keep $V_{T}$ constant and non-zero. UGAS of $\mathcal{A}_{T}$ by applying the nonsmooth invariance principle in [16], also using the well posedness result established at the beginning of the proof.

Proof of item 4. Item 4 follows straightforwad recalling Property 1 , the minimum dwell time converges to zero as $T$ goes to zero. Consequently, set $\mathcal{A}_{T}$ in (35) shrinks to $\mathcal{A} \times\{0\}$ as $T$ goes to zero, and since we prove in item 2 that $\mathcal{A}_{T}$ exists, we can make $\mathcal{A}_{T}$ arbitrarily close to $\mathcal{A} \times\{0\}$ by selecting $T$ sufficiently small. 
Remark 6. Note that all solutions to the hybrid dynamical system (34) present a minimum dwell-time in the ordinary time, given by T. This minimum dwell-time is not constant. Indeed, it increases if the solution does not satisfy any condition of $\mathcal{D}_{T}$. More precisely, the dwell-time is managed by $\eta$ and eventually by $T$, if solutions are far from the attractor $\mathcal{A}_{T}$. In other words, if $\tilde{x}$ is far from 0 , then the rule $\tilde{x}^{T} P(A \tilde{x}+B v) \geq-\eta \tilde{x}^{T} Q \tilde{x}$ and the rule $\tau \in[T, 2 T]$ induce the dwell time. On the other hand, the dwell-time is mainly managed by $T$, if solutions are close to the attractor $\mathcal{A}_{T}$; that means, if solutions are close to $\tilde{x}=0$, then the rule $\tau \in[T, 2 T]$ mainly induces the dwelltime.

The implementation of the hybrid system $\mathcal{H}_{T}$ follows the procedure given in Remark 4.

\section{Simulation}

Table I: Simulation parameters

\begin{tabular}{|c|c|c|c|}
\hline Parameter & Convention & Value & Units \\
\hline DC input voltage & $V_{\text {in }}$ & 48 & $V$ \\
Reference peak voltage & $V_{\max }$ & $120 \sqrt{2}$ & $V$ \\
Nominal angular frequency & $\omega$ & $2 \pi(60)$ & $\mathrm{rad} / \mathrm{s}$ \\
Inductor & $L$ & 50 & $\mathrm{mH}$ \\
Output capacitor & $C_{0}$ & 140.72 & $\mu F$ \\
Load resistance & $R_{0}$ & 240 & $\Omega$ \\
Estimated series resistance & $R_{L S}$ & 1.5 & $\Omega$ \\
\hline
\end{tabular}

In this section, we validate in simulation the hybrid controller designed in this paper for the inverter given in (1). From parameters given in Table I and from (3) and (4) we got the following desired behaviour

$$
x_{d}=\left[\begin{array}{c}
i_{L_{d}} \\
v_{c_{d}}
\end{array}\right]=\left[\begin{array}{c}
9 \sin \left(2 \pi 60 t+86^{\circ}\right) \\
120 \sqrt{2} \sin (2 \pi 60 t)
\end{array}\right],
$$

where it was applied the trigonometric relationship: $a \cos (x)+$ $b \sin (x)=\sqrt{\left(a^{2}+b^{2}\right)} \sin \left(x+\operatorname{atan}\left(\frac{b}{a}\right)\right)$. Moreover, we take $\rho=1000$ and $P=\left[\begin{array}{cc}24.71 & 0.10 \\ 0.10 & 0.07\end{array}\right] \cdot T^{*}$ is computed considering a chartering of $i_{C}$ equal to $2 \mathrm{~A}$ and a chattering of $v_{C}$ equal to $10 \mathrm{~V}$, giving $T^{*}=0.041$.

First, note that condition (15) is satisfied. Moreover, from (1), (3), (4) and the convex combination $u=\lambda_{1}-\lambda_{-1}$ with $\lambda_{1}+\lambda_{-1}=1$, we can achieve $x=x_{d}$ with $\lambda_{1, e}=0.5+$ $0.003 \sin (2 \pi 60 t)+0.34 \cos (2 \pi 60 t)$, satisfying condition (16).

Some simulations for different initial conditions are performed in MATLAB/Simulink by using the HyEQ Toolbox [17]. We show the average total jump count (the average is performed from different initial conditions) with respect to $\eta$ and $T$ in Fig. 3, for the transient time, and Fig. 4, for the steady state. The systems is considered to be in steady state when the trajectory evolves within a $5 \%$ band. Note that in the steady state, $T$ mainly induces the main jump reduction. On the other hand, in the transient time slot, the jump reduction is managed by $\eta$ and $T$. Moreover, note that the jumps converge to infinity as $T$ goes to zero and $\eta$ goes to 1 (as noted in Remark 3). In practice, this is not desirable in terms of energy efficiency and reliability, since every switch dissipates energy and reduces the switch lifespan. On the other side, Fig. 5 shows the average normalized switching frequency and cost function $J$ (the normalization of these quantities is performed dividing by the corresponding maximum value obtained in simulations and, the average is performed from different initial conditions) in the transient time slot as a function of $\eta$ and for different $T$. Note that, the cost function is reduced with larger values of $\eta$, as noted in Remark 5. On the contrary, the trend of the switching frequency is to grow with larger values of $\eta$. Thus we may give up a little on the LQR performance level and suitably adjust the switching frequency, finding a satisfactory tradeoff solution between performance and switching frequency for different $T$, which corresponds to the intersection between the switching curve and the cost function. Finally, in Fig.6, we show the voltage and current error in steady state for different values of $T$. Note that the chattering between two jumps are smaller than the chattering given for computing $T^{*}$ above. We remark that the error, and consequently the chattering, is reduced as $T$ goes down, as given in Theorem 5, item 3. All these simulations show the noted in Remark 6.

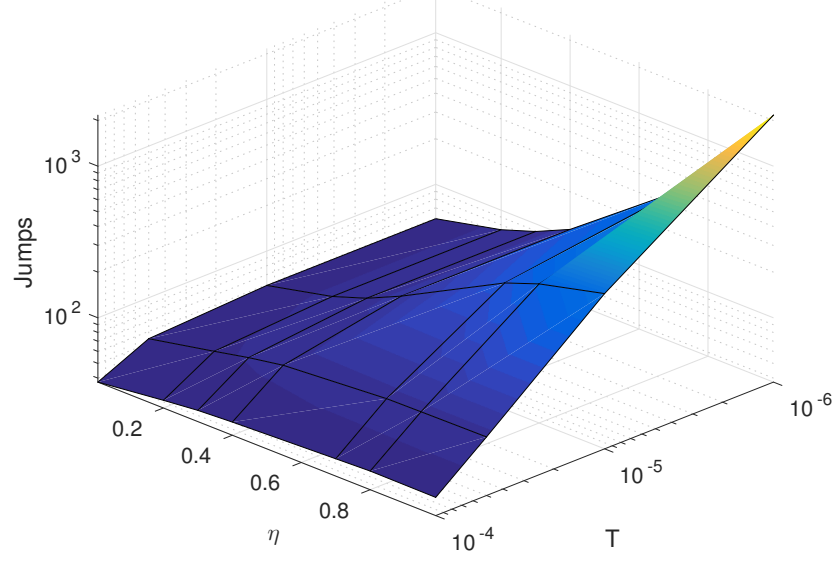

Figure 3: Jumps evolution w.r.t. $\eta$ and $T$ in transient time.

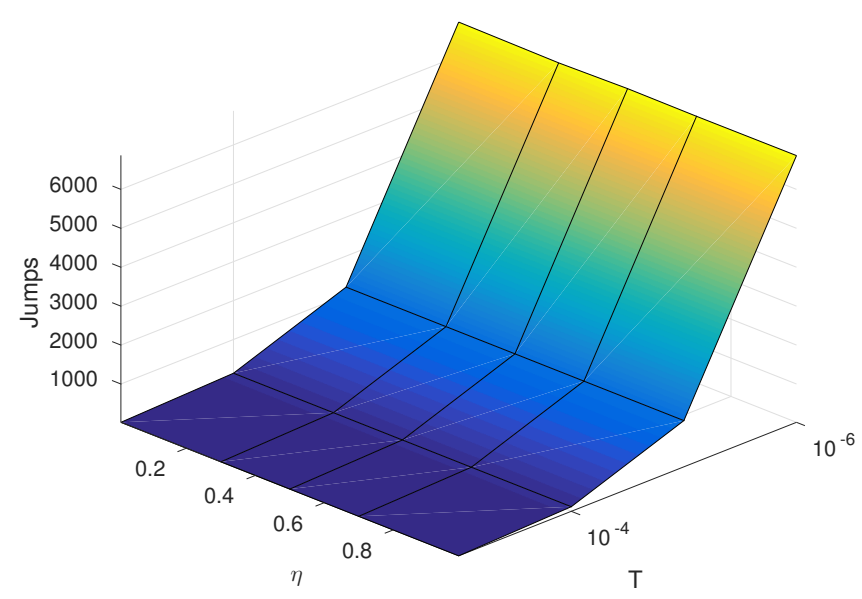

Figure 4: Jumps evolution w.r.t. $\eta$ and $T$ in the steady state. 

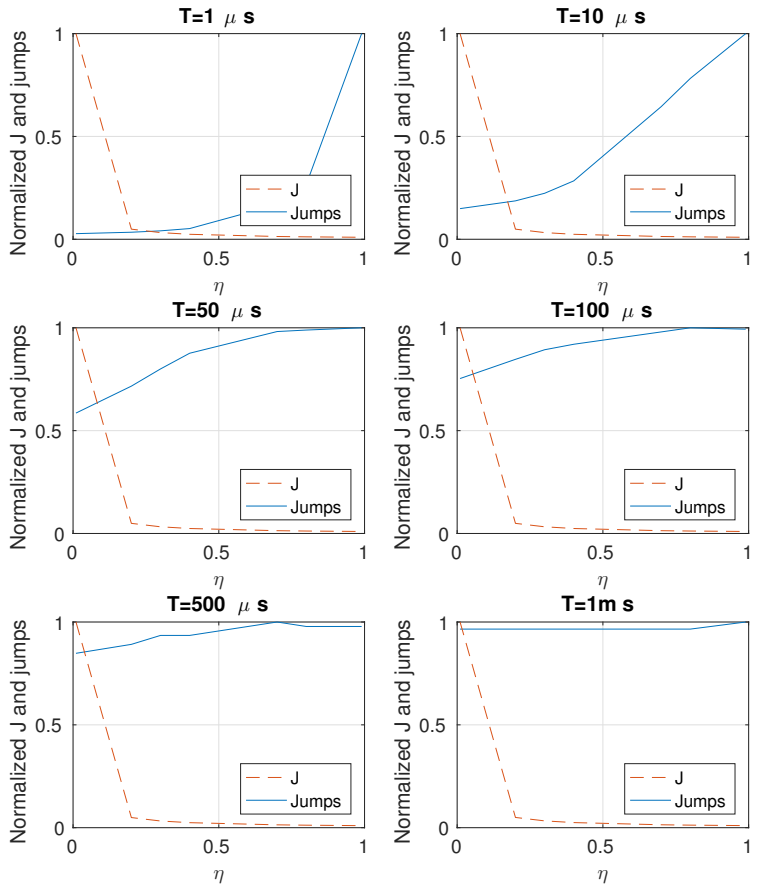

Figure 5: Average normalized jumps (solid) and cost function $J$ (dashed) s evolutions for different $T$.
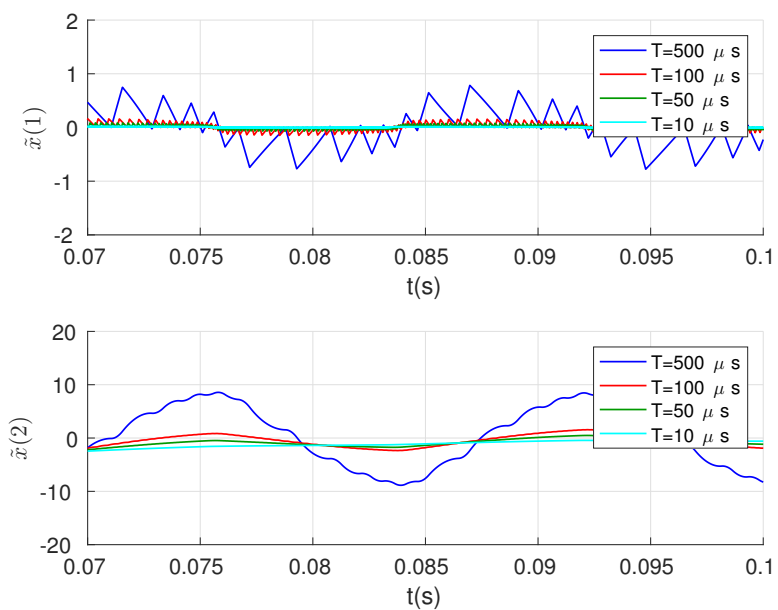

Figure 6: Evolution of current error, $\tilde{x}(1)$, and voltage error, $\tilde{x}(2)$, for different $T$.

Figure 7 shows the voltage and current evolutions of system (17)-(20) for $T=10 \mu s$ and with different $\eta$. This figure shows UGAS property of the attractor (22), which is guaranteed by Theorem 1. In addition, Fig. 8 performs a zoom of the jumps in the transient time marked in Fig. 7 with a shaded area. Once again, these figures show Remark 3, that states that, as $\eta$ grows to 1, we get arbitrary faster and faster switching.

Some simulations are given in Fig. 9 with system (17)-(20) with $\eta=0.1$ and different $T$. And a zoom of the switching in the steady state (marked in Fig. 9 with a shaded area) is given
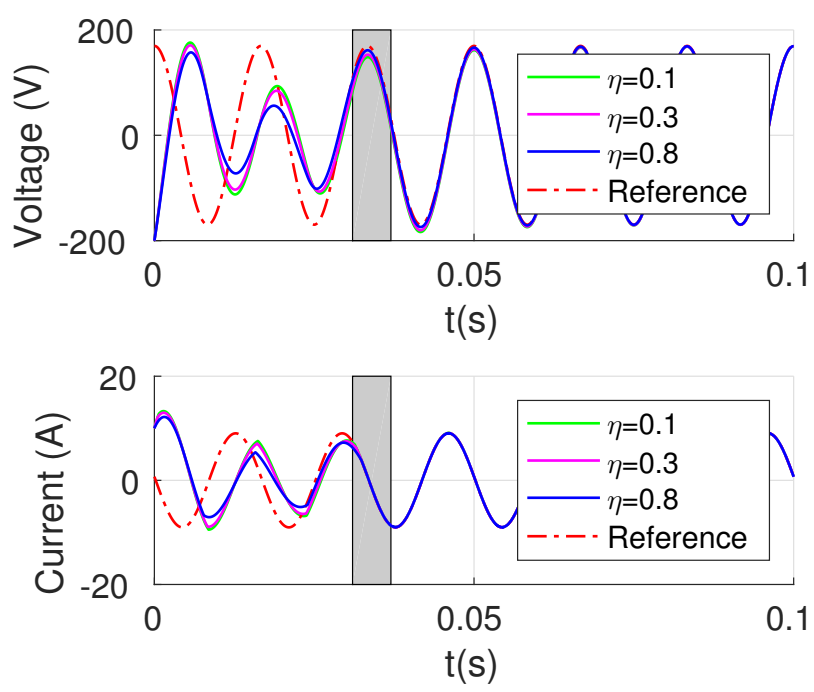

Figure 7: Inverter voltage and current evolutions with $T=$ $10 \mu s$.

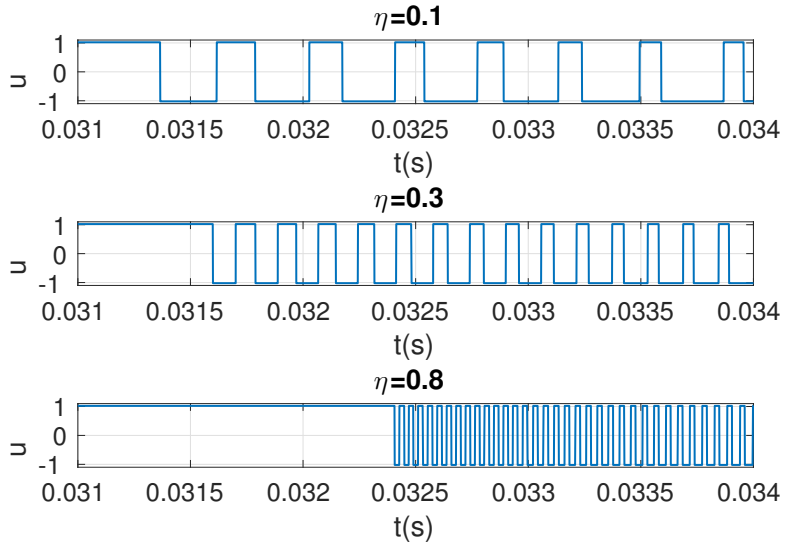

Figure 8: Zoom for $u$ in the inverter with $T=10 \mu \mathrm{s}$.

in Fig. 10. Note, as $T$ increases, it is expected a reduction of the switching frequency, which is consistent with Fig. 10. These simulations show the dwell-time and UGAS property for attractor $\mathcal{A}_{T}$ properties given in Theorem 3, item 1 and item 2 , respectively.

\section{EXPERIMENTAL RESULTS}

The hybrid control scheme is now tested in a $150 \mathrm{~W}$ halfbridge prototype. This prototype is composed by:

- 2 MOSFET AOT15S60 triggered using 2 photo-coupled drivers TLP350 and 1 IRS2004 driver, which receives only 1 control signal input, $u$, and generates the 2 gate signals with dead time;

- 2 sensors: an isolated closed-loop hall-effect transducer LV-20P for measuring the output voltage and an isolated closed-loop hall-effect transducer CAS 15-NP for measuring the inductor current. Outputs of both sensors are conditioning using analogue circuitry. 

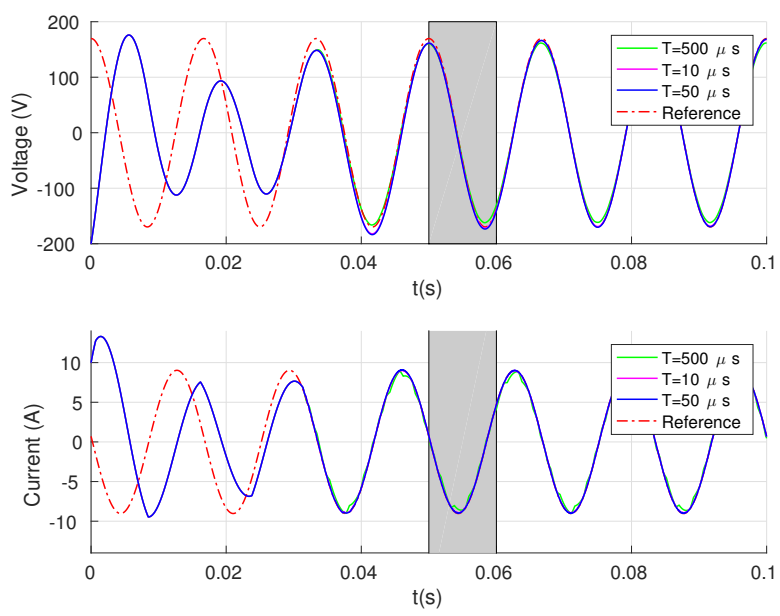

Figure 9: Voltage and current evolutions of the inverter and $\eta=0.1$.

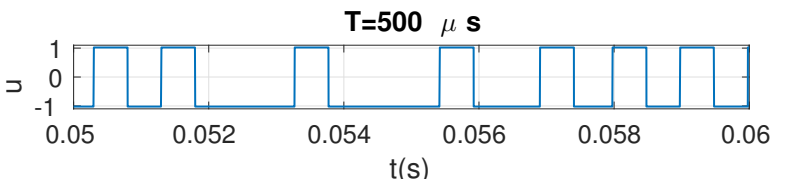

$\mathbf{T}=\mathbf{1 0 0} \mu \mathrm{s}$

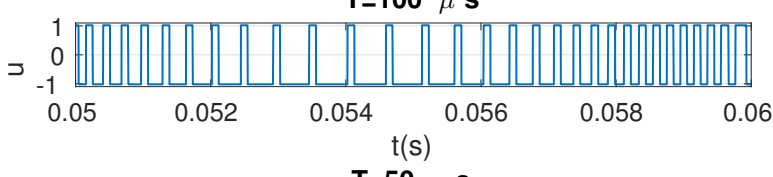

$\mathbf{T}=\mathbf{5 0} \mu \mathbf{s}$

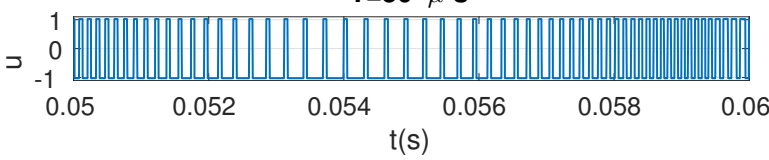

Figure 10: Zoom for $u$ in the inverter with $\eta=0.1$.

- 1 Digital Signal Processor (DSP) TMS32028335, which lets embedding the hybrid control algorithm, running with an internal sampling frequency of $100 \mathrm{kHz}$;

- 1 oscilloscope Tektronix MSO2014B;

- conventional voltage probes;

- 1 differential voltage probe;

- 1 isolated current probe TCP0030A;

- 1 programmable DC source BK precision XLN6024, which is used to provide the converter input voltage and

- 1 power source BK Precision 1672 feeding the sensors and auxiliary circuitry.

Figure 11 shows the prototype assembled with the measurement set-up.

The converter parameters appear in Table I and the desired behaviour in (43). The gains introduced by the sensors in the measurements (which can condition the circuit) are compensated into the DSP algorithms. Then, the hybrid scheme (34) with matrices $P$ and $Q$ given above are implemented in this DSP, and it is configured to have an internal interruption defining the sampling time in $10 \mu \mathrm{s}$. This time is used to generate a minimum dwell-time.

\section{A. Performance evaluation for different values of $\eta$}

First, we experimentally validate the efficiency of the hybrid scheme (34) w.r.t. $\eta$, which conditions the transient time, as shown in Fig. 7. For this one, several experimental tests were performed in the prototype in nominal conditions of input voltage and load, for $\eta=\{0.1,0.3,0.8\}$ and $T=10 \mu \mathrm{s}$. Note that these $\eta$ values are according simulation results Fig. 7. As shown in Fig. 12, system reaches the steady state in frequency, phase and amplitude before $50 \mathrm{~ms}$. The signal $s(t)$ is obtained from a digital output of the DSP and represents the polarity of the internally generated sinusoidal reference for $v_{C}$ ( $3 \mathrm{~V}$ if signal is positive and $0 \mathrm{~V}$ if signal is negative). Remark that the switching frequency decreases as $\eta$ diminishes, as is shown in simulation (see Fig. 8).

\section{B. Steady-state performance for different values of $T$}

Now, we validate the hybrid scheme (34) for different minimum dwell times in steady state, $T=\{50 \mu, 100 \mu, 500 \mu s\}$ and $\eta=0.1$, applying several tests in nominal conditions. Figure 13 shows the steady state behaviour for different $T$. We highlight that the voltage and current output are practically the same despite different $T$. However, the switching are reduced, as expected. These results show the dwell-time and UGAS property for attractor $\mathcal{A}_{T}$ properties given in Theorem 3, item 1 and item 2, respectively. Moreover, we measured the THD of the output voltage using a Power Quality Analyzer Fluke 43B with constrained computations to 21 harmonics $(1.26 \mathrm{kHz})$. THD values were obtained in a range between $1.0 \%$ and $1.3 \%$, presenting the best result for $\eta=0.1$ and $T=100 \mu s$ (see Fig. 14.c). In order to complement this result, a FFT analysis with extended bandwidth was performed using PSIM simulations for the same values of $\eta$ and $T$ obtaining THD values in a range between $1.8 \%$ and $3.5 \%$ and presenting the best result for $\eta=0.1$ and $T=100 \mu \mathrm{s}$. This means that for higher values of $T$, it is expected that THD increases due to the absence of commutations during a relevant slot time. This fact is very important for low performance devices.

Moreover, we measured the inverter $\mathrm{THD}^{1}$, whose values are obtained in a range between $1.0 \%$ and $1.3 \%$ using a Power Quality Analizer Fluke 43B. The best measurement was obtained for $\eta=0.1$ and $T=100 \mu s$ (see Fig.14 c). This means that for higher $T$ or smaller $\eta$ the THD increases due to the absence of switching during a relevant slot time. This fact is very important for low-cost devices, i.e. for low performance devices.

\section{CONCLUSIONS AND FUTURE WORK}

In this work, we proposed a controller following a HDS framework for a dc-ac converter composed of continuous variables (current and voltage) and discrete variables (state of switches), whose main problem is to track a sinusoidal reference. The main advantage of this method is to manage

\footnotetext{
${ }^{1}$ The Total Harmonic Distortion (THD) is an output signal corresponding to a quality indicator of electronic converters
} 


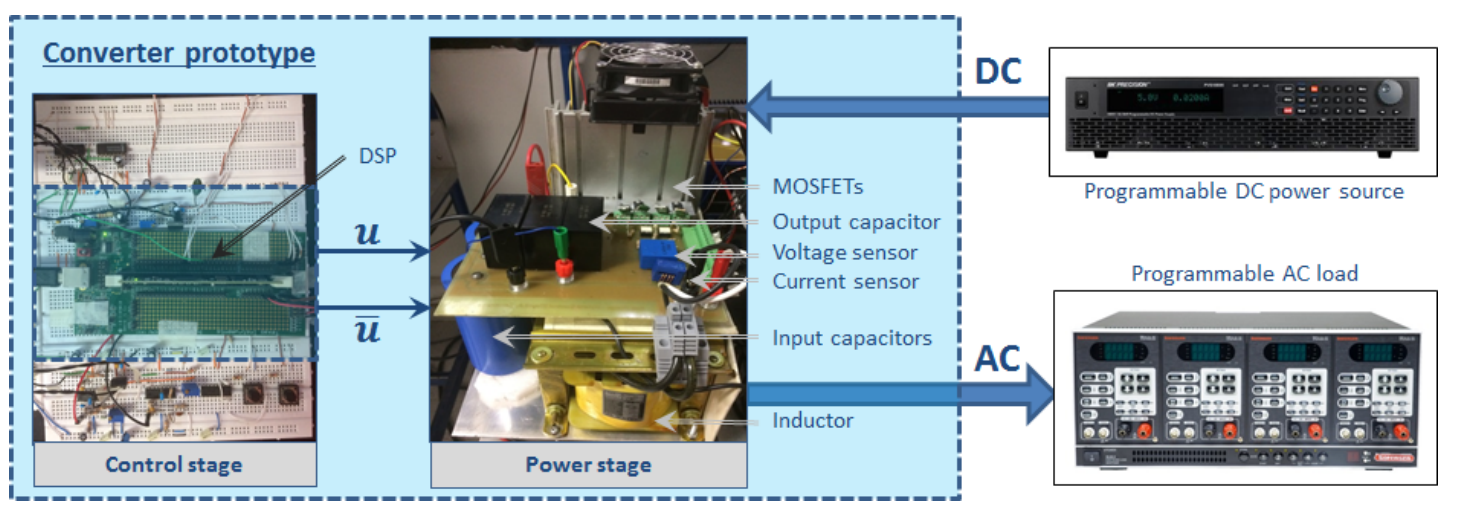

Figure 11: Laboratory prototype and experimental set-up

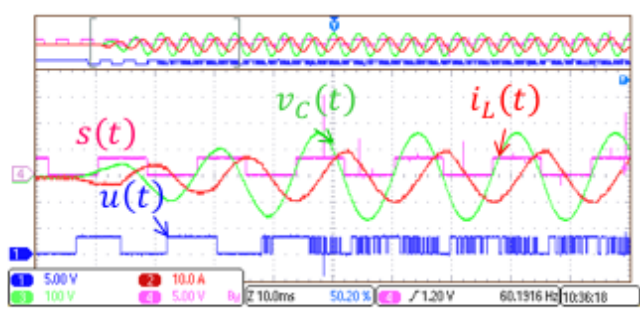

a)

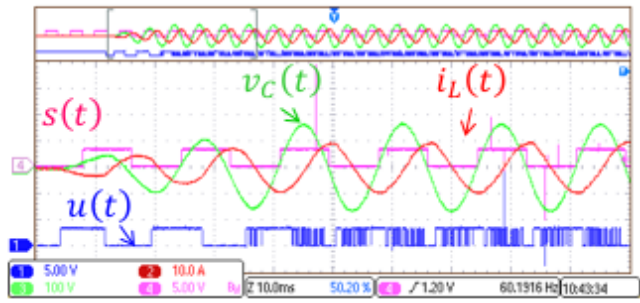

c)

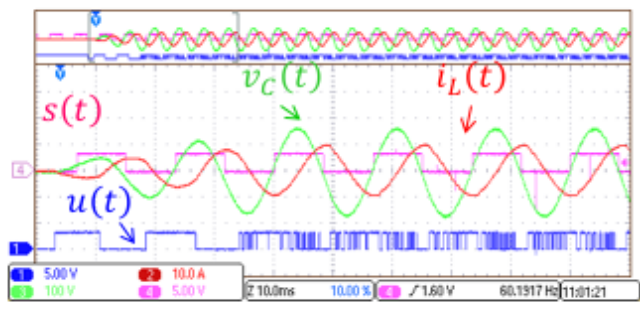

e)

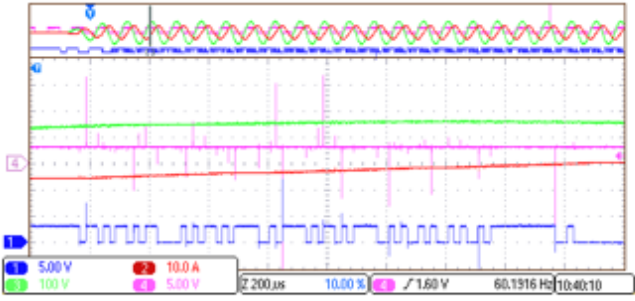

b)

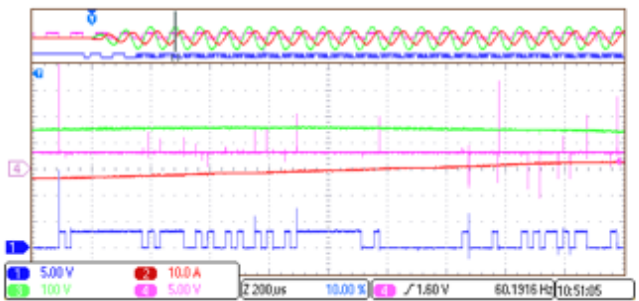

d)

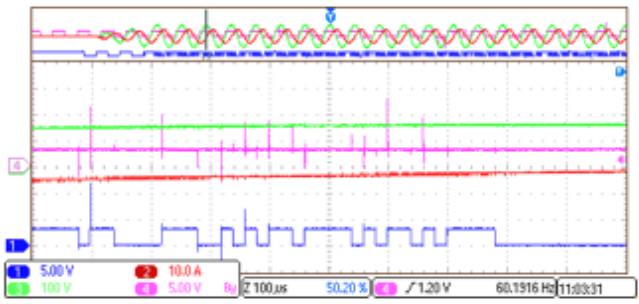

f)

Figure 12: Oscilloscope captures of the inverter signals (input, $s$, and outputs, $v_{C}$ and $i_{L}$ ) during transient-state and zoom of $u$ for $T=10 \mu s$ and $\eta=0.8 \mathrm{a}$ )-b), $\eta=0.3 \mathrm{c}$ )-d) and $\eta=0.1 \mathrm{e}$ )-f).

the switching frequency in both transient and steady states. We showed practical results using a time-regularization that allows: 1) to stabilise set compact set $\mathcal{A}_{T}$ being $T$ small enough to adjust the steady state switching frequency, 2) to get a positive minimum dwell-time in each mode, $T$, whose size can be modulated by $\eta$ and 3) to deal with a tradeoff between dwell-time on the one hand and an LQR performance level, tuning parameter $\eta$ and the size of the asymptotically stable set around the desired operating point, tuning parameter $T$, on the other hand. Some experiments validate our proposed hybrid controller.

A future work is to use this inverter to inject energy in the power grid, which means to synchronize the inverter in amplitude, frequency and phase with the power grid. Likewise, it will be desirable to ensure that the output voltage is robust with respect to load and DC/AC converter parameter variations.

\section{REFERENCES}

[1] A. Antonopoulos, L. Angquist, and H. Nee, "On dynamics and voltage control of the modular multilevel converter," in IEEE European Conference on Power Electronics and Applications (EPE). IEEE, 2009, pp. 3353-3362.

[2] R. Middlebrook and S. Cuk, "A general unified approach to modelling switching-converter power stages," in IEEE Power Electronics Specialists Conference. IEEE, 1976, pp. 18-34.

[3] H. Sira-Ramírez and R. Silva-Ortigoza, Control design techniques in power electronics devices. Springer Science \& Business Media, 2006. 


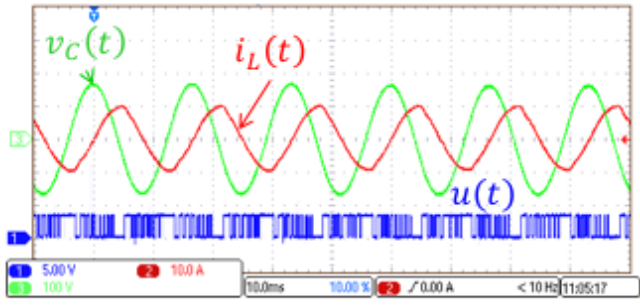

a)

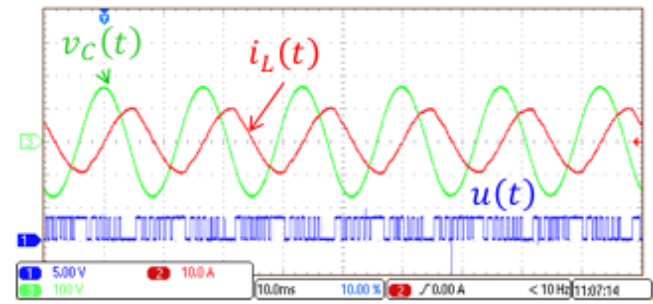

c)

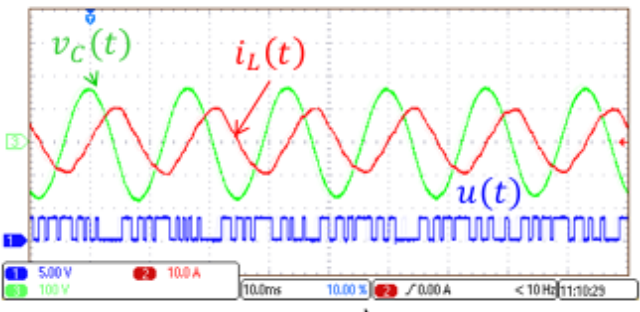

e)

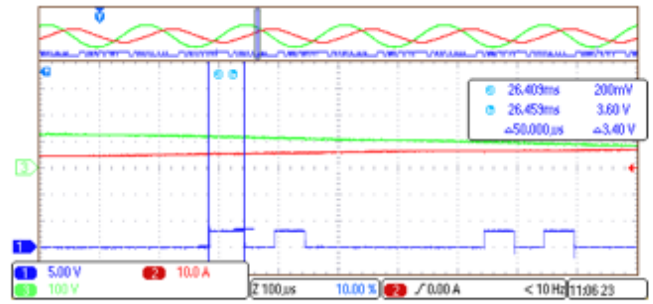

b)

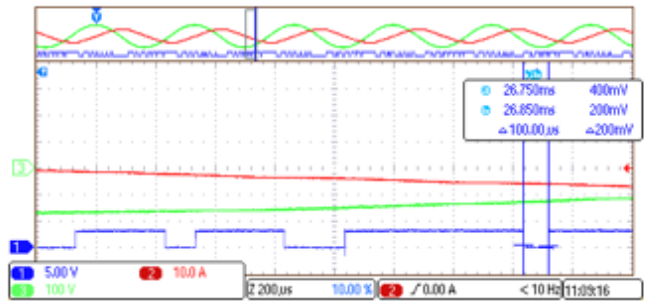

d)

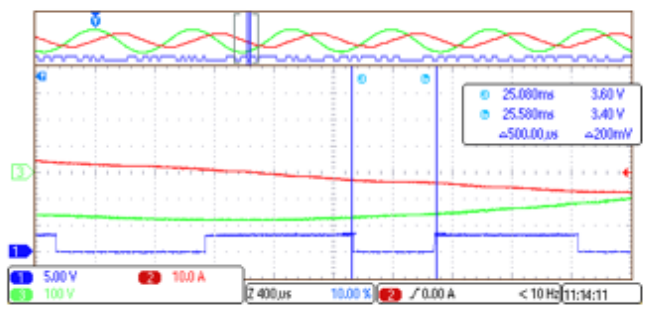

f)

Figure 13: Oscilloscope captures of the inverter signals (input, $s$, and outputs, $v_{C}$ and $i_{L}$ ) in the steady state and zoom of $u$ for $\eta=0.1$ and $T=50 \mu s$ a)-b), $T=100 \mu s \mathrm{c}$ )-d) and $T=500 \mu s$ e)-f).
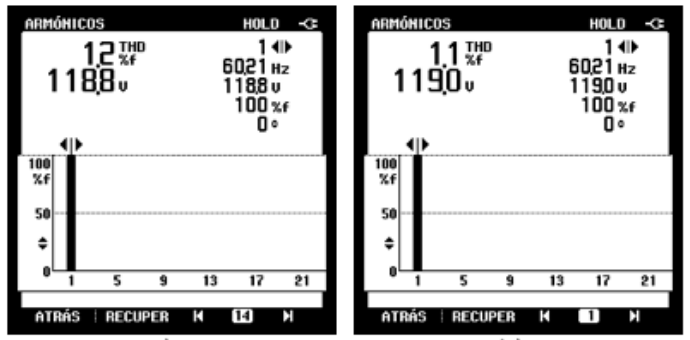

a)

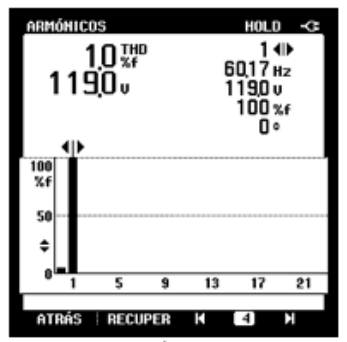

c)

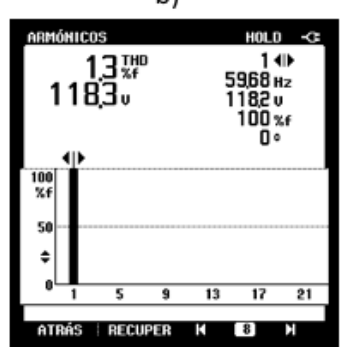

d)

Figure 14: THD measurements: a) $\eta=0.1$ and $T=10 \mu \mathrm{s}$; b) $\eta=0.1$ and $T=50 \mu s ;$ c) $\eta=0.1$ and $T=100 \mu s ;$ and d) $\eta=0.1$ and $T=500 \mu s$

[4] M. Senesky, G. Eirea, and T. J. Koo, "Hybrid modelling and control of power electronics," in International Workshop on Hybrid Systems: Computation and Control. Springer, 2003, pp. 450-465.

[5] C. Sreekumar and V. Agarwal, "A hybrid control algorithm for voltage regulation in dc-dc boost converter," IEEE Trans. on Industrial Elec- tronics, vol. 55, no. 6, pp. 2530-2538, 2008.

[6] G. S. Deaecto, J. C. Geromel, F. Garcia, and J. Pomilio, "Switched affine systems control design with application to DC-DC converters," IET control theory \& applications, vol. 4, no. 7, pp. 1201-1210, 2010.

[7] C. Albea Sanchez, G. Garcia, and L. Zaccarian, "Hybrid dynamic modeling and control of switched affine systems: application to dc-dc converters," in proc. IEEE Conference on Decision and Control (CDC), 2015, pp. 2264-2269.

[8] J. Chai and R. G. Sanfelice, "A robust hybrid control algorithm for a single-phase dcac inverter with variable input voltage," in proc. IEEE American Control Conference (ACC), 2014, pp. 1420-1425.

[9] L. Torquati, R. Sanfelice, and L. Zaccarian, "A hybrid predictive control algorithm for tracking in a single-phase dcac inverter," in proc. IEEE Control Technology and Applications (CCTA), 2017, pp. 904-909.

[10] C. Albea Sanchez, O. Santos, D. Z. Prada, F. Gordillo, and G. Garcia, "Hybrid control scheme for a half-bridge inverter," IFAC Proceedings Volumes, vol. 50, no. 1, pp. 9336-9341, 2017.

[11] B. A. Francis, "The linear multivariable regulator problem," SIAM Journal on Control and Optimization, vol. 15, no. 3, pp. 486-505, 1977.

[12] R. Goebel, R. Sanfelice, and A. Teel, Hybrid Dynamical Systems: modeling, stability, and robustness. Princeton University Press, 2012.

[13] D. Liberzon and A. Morse, "Basic problems in stability and design of switched systems," IEEE Control Systems Magazine, vol. 19, no. 5, pp. 59-70, 1999.

[14] C. Prieur, A. R. Teel, and L. Zaccarian, "Relaxed persistent flow/jump conditions for uniform global asymptotic stability," IEEE Trans. on Automatic Control, vol. 59, no. 10, pp. 2766-2771, October 2014.

[15] M. Corless and A. Frazho, Linear systems and control: an operator perspective. CRC Press, 2003.

[16] A. Seuret, C. Prieur, S. Tarbouriech, A. Teel, and L. Zaccarian, "A nonsmooth hybrid invariance principle applied to robust event-triggered design," Submitted to IEEE Transactions on Automatic Control. See also: https://hal.archives-ouvertes.fr/hal-01526331/.

[17] R. G. Sanfelice, D. Copp, and P. A. Nanez, "A toolbox for simulation of hybrid systems in Matlab/Simulink: Hybrid equations (HyEQ) toolbox," in Hybrid Systems: Computation and Control Conference, 2013. 